Pacific Journal of Mathematics

INVARIANT EXTENSION OF LINEAR FUNCTIONALS 


\title{
INVARIANT EXTENSION OF LINEAR FUNCTIONALS
}

\author{
V. L. KLEE, JR.
}

1. Introduction. The expression $[L, \mathcal{J}, f, p]$ will mean (i) $L$ is a real linear space and $\mathcal{F}$ a set of linear transformations of $L$ into $L$; (ii) $f$ is a linear functional on a linear subspace $D_{f}$ of $L$; (iii) $p$ is a positively homogeneous subadditive functional on $L$; (iv) $f \leqq p ;(\mathrm{v}) T D_{f} \subset D_{f}$ and $f T=f$ for each $T \in \mathcal{J}$. If $[L, \mathcal{J}, f, p]$, then $\langle L, \mathcal{J}, f, p\rangle$ will denote the set of all $F$ such that $[L, \mathcal{J}, F$, $p$ ], $D_{F}=L$, and $F \mid D_{f}=f$. With $I$ denoting the identity transformation on $L$, the Hahn-Banach theorem $[2, \mathrm{p} .28]$ asserts that if $[L,\{I\}, f, p]$, then $\langle L,\{I\}, f$, $p\rangle$ is nonempty. More general conditions have been obtained by Agnew and Morse [1] and Woodbury [10] under which $\langle L, J, f, p\rangle$ is nonempty, and by Dunford [3] and Yood [11] under which $\langle L, \mathcal{J}, f, m p\rangle$ includes for some $m \geqq 1$ an $F$ which is not identically zero. Their results have applications to the extension and existence of measures $[1 ; 3 ; 10 ; 11]$, limits, and so on $[1]$, and in proving the "normality" (as used in connection with the Banach-Tarski paradox) of certain sets [6]. We prove here a theorem whose corollaries include an extension of the results of Agnew and Morse and Woodbury, and also include the principal results of Dunford and Yood, although Yood's work with relaxed boundedness conditions is not covered here. In addition to the cases in which $\mathcal{J}$ is a commutative semigroup or a finite or solvable group, we are able to handle the case in which $\mathcal{J}$ is a compact group of bounded linear transformations.

2. The theorem. We shall use the following result.

(2.1) Lemma. Suppose L, J, f, and p satisfy conditions (i)-(iii), and for each $x \in L$ let

$$
q(x)=\inf \left\{p\left(x+\sum_{1}^{k} T_{i} y_{i}\right) \mid k \text { a positive integer, } T_{i} \in \mathcal{J}, y_{i} \in L\right\} .
$$

Then $f \leq q$ on $D_{f}$ if and only if there exists $F \in\langle L,\{I\}, f, p\rangle$ such that $F T \equiv 0$ on $L$ for each $T \in \mathcal{J}$.

Received January 16, 1953.

Pacific J. Math. 4 (1954), 37-46 
Proof. If $F$ is as described, $x \in D_{f}, T_{i} \in \mathcal{J}$, and $y_{i} \in L$, then

$$
f(x)=F(x)=F\left(x+\sum_{1}^{k} T_{i} y_{i}\right) \leqq p\left(x+\sum_{1}^{k} T_{i} y_{i}\right) \text {, }
$$

and thus $f \leqq q$ on $D_{f}$. Now suppose conversely that $f \leqq q$ and note first that for arbitrary $x \in L_{,}, T_{i} \in \mathcal{J}$, and $y_{i} \in L$,

$$
0=f(0) \leqq q(0) \leqq p\left(\sum_{1}^{k} T_{i} y_{i}\right) \leqq p(-x)+p\left(x+\sum_{1}^{k} T_{i} y_{i}\right) \text {, }
$$

whence $q(x) \geqq-p(-x)$ and $q$ is everywhere finite-valued. Clearly $q$ is positively homogeneous. Furthermore, if $x$ and $x^{\prime}$ are points of $L$ and $\epsilon>0$ there are transformations $T_{i}, T_{i}^{\prime}$ in $Y$ and points $y_{i}, y_{i}^{\prime}$ in $L$ such that

$$
p\left(x+\sum_{1}^{k} T_{i} y_{i}\right) \leqq q(x)+\epsilon,
$$

and similarly for $x^{\prime}$. But then

$$
\begin{aligned}
q\left(x+x^{\prime}\right) & \leqq p\left(x+x^{\prime}+\sum_{1}^{k} T_{i} y_{i}+\sum_{1}^{k^{\prime}} T_{i}^{\prime} y_{i}^{\prime}\right) \\
& \leqq p\left(x+\sum_{1}^{k} T_{i} y_{i}\right)+p\left(x^{\prime}+\sum_{1}^{k^{\prime}} T_{i}^{\prime} y_{i}^{\prime}\right) \\
& \leqq q(x)+q\left(x^{\prime}\right)+2 \epsilon .
\end{aligned}
$$

Thus $q$ is subadditive, and since $f \leqq q$ the Hahn-Banach theorem quarantees the the existence of an $F \in\langle L,\{I\}, f, q\rangle \subset\langle L,\{I\}, f, p\rangle$. For $T \in \mathcal{J}$ and $z \in L$ we have

$$
F(T z) \leqq p(T z+T(-z))=p(0)=0 .
$$

Similarly, $F(T(-z)) \leqq 0$, so $F$ has the desired properties and the proof is complete.

(2.2) THEOREM. If $\left[L, \mathcal{J}_{,} f, p\right]$, then the statements $(\alpha)-(\gamma)$ below are equivalent. If $[L, \mathcal{J}, f, p]$ and $\mathcal{J}$ is a semi-group, then $(\alpha)-(\delta)$ are equivalent: 
( $\alpha)\langle L, \mathcal{J}, f, p\rangle$ is nonempty;

( $\beta$ ) for each finite $\& \subset \mathcal{J},\langle L$, \& $f, p\rangle$ is nonempty;

$(\gamma)$ if $x \in D_{f}, T_{i} \in \mathcal{J}$, and $y_{i} \in L$, then $f(x) \leqq p\left(x+\sum_{1}^{k}\left(T_{i}-I\right) y_{i}\right)$;

( $\delta$ ) there exists $g \in\langle L,\{I\}, f, p\rangle$ such that $g S T=g T S$ and $g T \leqq p$ whenever $S, T \in J$.

Proof. Since $F \in\langle L, j, f, p\rangle$ if and only if $F \in\langle L,\{l\}, f, p\rangle$ and $F R \equiv 0$ for each $R \in J-I$, from the lemma it follows at once that $(\alpha)$ and $(\gamma)$ are equivalent, and from this it follows that they are both equivalent to $(\beta)$. Now if $F \in\langle L, J$, $f, p\rangle$ and $S, T \in \mathcal{\prime}$, then $F S T=F=F T S$ and $F T=F \leqq p$, so $(\alpha) \operatorname{implies}(\delta)$. We complete the proof by showing that if $[L, g, f, p]$ and $g$ is a semi-group, then $(\delta)$ implies $(y)$.

Consider arbitrary $x \in D_{f}, T_{i} \in \mathcal{r}, y_{i} \in L$, and let

$$
\epsilon=p\left(x+\sum_{1}^{k}\left(T_{i}-I\right) y_{i}\right)-f(x)
$$

We wish to prove $\epsilon \geqq 0$. Let $\Phi_{n}$ be the set of all functions on $\{1, \cdots, k\}$ to $\{0, \cdots, n\}$; and, for $1 \leqq i \leqq k$, let $\Phi_{n, i}$ be the set of all $\phi \in \Phi_{n}$ for which $\phi(i)=$ 0 . For $\phi \in \Phi_{n}$ let

$$
S_{\phi}=T_{1}^{\phi(1)} T_{2}^{\phi(2)} \ldots T_{k}^{\phi(k)}
$$

Define

$$
A=\sum_{i=1}^{k} \sum_{\phi \in \Phi_{n}} g S_{\phi}\left(T_{i}-I\right) y_{i}
$$

Now $g$ is linear, $g=f$ on $D_{f}, g S_{\phi} \leqq p$ on $L$, and $f S_{\phi}=f$ on $D_{f}$, so we have

$$
A=\sum_{\phi \in \Phi_{n}} g S_{\phi}\left[x+\sum_{1}^{k}\left(T_{i}-I\right) y_{i}\right]-\sum_{\phi \in \Phi_{n}} f S_{\phi} x \leqq(n+1)^{k} \epsilon .
$$

But $\mathcal{J}$ is a semi-group and $g S T=g T S$ for $S, T \in \mathcal{J}$, so the terms $S_{\phi} T_{i} y_{i}$ for $\phi(i) \neq n$ and $S_{\phi}(-I) y_{i}$ for $\phi(i) \neq 0$ all cancel out, and we have

$$
A=\sum_{i=1}^{k} \sum_{\phi \in \Phi_{n, i}} g S_{\phi}\left(T_{i}^{n+1}-I\right) y_{i} .
$$


Now since $g T \leqq p$ for all $T \in \mathcal{J}$,

$$
g S_{\phi}\left(T_{i}^{n+1}-l\right) y_{i}=-\left[g S_{\phi} T_{i}^{n+1}\left(-y_{i}\right)+g S_{\phi} y_{i}\right] \geqq-\left[p\left(-y_{i}\right)+p\left(y_{i}\right)\right]
$$

Hence

$$
A \geqq-\sum_{i=1}^{k} \cdot \sum_{\phi \in \Phi_{n, i}}\left[p\left(-y_{i}\right)+p\left(y_{i}\right)\right]=(n+1)^{k-1} B,
$$

with

$$
B=-\sum_{1}^{k}\left[p\left(-y_{i}\right)+p\left(y_{i}\right)\right] .
$$

Thus $B \leqq(n+1) \in$ for each $n$, and since $B$ is finite and independent of $n$ this implies that $\epsilon \geqq 0$, completing the proof.

The expression $[L, \mathfrak{J}, f, p]_{b}$ will mean that $[L, \mathfrak{J}, f, p], b \geqq 1$, and $\mathcal{J}$ is $p$ bounded with bound $b$, that is, $p T \leqq b p$ for each $T \in \mathcal{J}$.

By using the lemma and the equivalence of $(\alpha)$ and $(\delta)$ in (2.2), one can prove that if $[L, J, f, p]_{1}$ and $J$ is a semi-group, then $(\alpha)-(\delta)$ are equivalent to

$(\epsilon)$ if $x \in D_{f}, S_{i} \in \mathfrak{J}, T_{i} \in \mathfrak{J}$, and $y_{i} \in L$, then

$$
f(x) \leqq p\left(x+\sum_{1}^{k}\left(S_{i} T_{i}-T_{i} S_{i}\right) y_{i}\right)
$$

3. The corollaries. The closure and convex hull of a set $X$ will be denoted by Cl $X$ and conv $X$ respectively.

(3.1) Corollary. Suppose $[L, \mathcal{J}, f, p]_{b}$ and either $b=1$ or $p \geqq 0$. Then each of the following implies that $\langle L, \mathcal{J}, f, b p\rangle$ is nonempty:

(a) I is a commutative semi-group;

(b) there is a linear transformation $R$ of $L$ into $L$ such that $R J=R, f R=f$, and $p R(z) \in \mathrm{Cl} p(\operatorname{conv} \zeta(z))$ for each $z \in L$;

(c) every finite subset of ${ }^{\text {J }}$ is contained in a finite subgroup of $\mathrm{J}$;

(d) $p$ is a norm for $L$ and $J$ is a group which is compact in the uniform topology of operators. 
Proof. For $b=1$ we have $p=b p$; and for $p \geqq 0, p \leqq b p$ ( since, as always, $b \geqq 1)$. Thus $[L, J, f, b p]$, and (2.2) is applicable.

(a) The Hahn-Banach theorem guarantees the existence of a

$$
g \in\langle L,\{l\}, f, p\rangle \subset\langle L,\{l\}, f, b p\rangle .
$$

Since $\mathcal{J}$ is commutative, $g S T=g T S$ for $S, T \in \mathcal{J}$. Furthermore, $g T \leqq p T \leqq b p$. Thus $(\delta)$ of $(2.2)$ is satisfied for $[L, J, f, b p]$, and the desired conclusion follows from (2.2).

(b) Let $R$ be as described. Then for $x \in D_{f}, T_{i} \in \mathcal{J}$, and $y_{i} \in L$, we have

$$
f(x)=f R(x) \leqq p R(x)=p\left(R x+\sum_{1}^{k}\left(R T_{i}-R\right) y_{i}\right)=p R(z),
$$

with

$$
z=x+\sum_{1}^{k}\left(T_{i}-1\right) y_{i}
$$

But $p R(z) \in \mathrm{Cl} p($ conv $\supset(z))$, so for each $\epsilon>0$ there are $S_{1}, \ldots, S_{n}$ in $J_{\text {and }}$ $t_{1}, \cdots, t_{n} \geqq 0$ with sum 1 such that $p R(z)$ differs by less than $\epsilon$ from $p S(z)$, with

$$
S=\sum_{1}^{n} t_{i} S_{i}
$$

Now

$$
p S(z) \leqq \sum_{1}^{n} t_{i} p S_{i}(z) \leqq \sum_{1}^{n} t_{i} b p(z)=b p(z) .
$$

Thus $f(x) \leqq b p(z)$, so $(y)$ of $(2.2)$ is satisfied and the desired conclusion follows from (2.2).

(c) In order to show that $\langle L, \mathcal{J}, f, b p\rangle$ is nonempty it will suffice, in view of $(\alpha)$ and $(\beta)$ in $(2.2)$, to show that (b) must hold if $\zeta$ is a finite group. Let $T_{1}, \cdots, T_{n}$ be the members of $g$ and $R=(1 / n)\left(T_{1}+\cdots+T_{n}\right)$. Then the last two conditions of (b) are clearly satisfied, and $\mathcal{J} T_{j}$ is merely a permutation of J, so $R T_{j}=R$ for each $j$. 
The proof of ( 3.1$)$ will be completed by showing that ( $d$ ) implies ( b), but we defer this until $\$ 4$.

(3.2) Cor Ollary. Suppose $[L, \mathfrak{J}, f, p]_{1}, \mathfrak{J}$ is a semi-group, and for some $x \in D_{f}, f(x)=p(x)$ and $p$ has a unique supporting functional at $x$ (that is, there is a unique linear functional $F \leqq p$ on $L$ such that $F(x)=p(x))$. Then $\langle L, j$, $f, p)$ is nonempty.

Proof. Consider an arbitrary $S \in \mathcal{J}$; $S$ generates in $\mathcal{J}$ a commutative semigroup $S^{*}$, so by $\left(3.1\right.$ ) (a) there must exist $F_{S} \in\left\langle L, S^{*}, f, p\right\rangle$. Now $F_{S} \leqq p$ and $F_{S}(x)=f(x)=p(x)$, so $F_{S}=F$. Thus we have

$$
F \in \bigcap_{S \in \mathcal{J}}\left\langle L, S^{*}, f, p\right\rangle=\langle L, \mathfrak{J}, f, p\rangle
$$

completing the proof.

(3.3) Corollary. Suppose $[L, \exists, f, p]_{b}$, either $b=1$ or $p \geqq 0$, and $j$ is a positive integer. Then each of the following implies that $\left\langle L, J, f, b^{j} p\right\rangle$ is nonempty:

(e) $J$ is a group and $\left\langle L, J^{(k)}, f, b^{j-k} p\right\rangle$ is nonempty for some integer $k$ with $0 \leqq k \leqq j$, where $\mathfrak{J}^{(k)}$ is the $k^{\text {th }}$ derived group of $\mathrm{J}$;

(f) $\mathcal{I}$ is a solvable group, with $\mathcal{J}^{(j)}=\{I\}$.

Proof (e). It suffices to show that if $1 \leqq k \leqq j$ and there exists $g \in\left\langle L, g^{(k)}\right.$, $\left.f, b^{j-k} p\right\rangle$, then $\left\langle L, g^{(k-1)}, f, b^{j-k+1} p\right\rangle$ is nonempty. Now for $S, T \in g^{(k-1)}$ we have $T S T^{-1} S^{-1} \in \mathcal{J}^{(k)}$, so

$$
g S T=g\left(T S T^{-1} S^{-1}\right) S T=g T S .
$$

Furthermore,

$$
g T \leqq b^{j-k} p T \leqq b^{j-k} b p=b^{j-k+1} p
$$

Thus it follows from (2.2) that $\left\langle L, J^{(k-1)}, f, b^{j-k+1} p\right\rangle$ is nonempty, and this completes the proof.

(f) If $J^{(j)}=\{I\}$, then it follows from the Hahn-Banach theorem that (e) holds with $k=j$.

(3.4) Corollary. Let $h$ be the linear functional with domain $D_{h}=\{0\}$. Suppose $[L, J, h, p], \mathcal{J}$ is $p$-bounded, $p>0$ on $L-\{0\}$, and there is an $x \in L-$ $\{0\}$ such that $T x=x$ for each $T \in J$. Then if $(\mathrm{a}),(\mathrm{c}),(\mathrm{d})$, or $(\mathrm{f})$ is satisfied, 
there exists an $m<\infty$ and a not-identically-zero $F \in\langle L, \mathrm{~J}, h, m p\rangle$.

Proof. For each real $t$, let $f(t x)=t p(x)$. Then $[L, J, f, p]$, so from $(3.1)$ and (3.3) it follows that $\langle L, J, f, m p\rangle$ is nonempty for sufficiently large $m<\infty$. Since $f$ is not identically zero, this completes the proof.

For the case in which $p$ is $\mathcal{J}$-invariant ( $p T=p$ for each $T \in \mathcal{J}),(3.1$ ) (a) was given by Woodbury [10], and (3.3) was proved by Agnew and Morse [1]. (3.4) (a), (c), and (f) were proved by Yood [11], who obtained his results under boundedness assumptions weaker than those employed here.

By an argument analogous to that of Agnew and Morse $[1$, p. 24-25] the following can be proved.

(3.5) Hy PотHESES: $X$ is a set; $m$ is a set of subsets of $X$ such that if $A$, $B \in m$, then $A \cup B \in m$ and $A-B \in m ; m$ is a finitely additive real-valued measure on $m ; y$ is a group of biunique transformations of $X$ onto $X$ which is either solvable or such that every finite subset is contained in a finite subgroup; $k_{T} \mid T \in \mathcal{J}$ is a positive-valued function such that $m T=k_{T} m$ for each $T \in \mathcal{J}$; \& is the set of all sets which are contained in some member of $M$.

CONCLUSION: There is a finitely additive real-valued measure $\mu$ on such that $\mu \mid \mathrm{m}=m$ and $\mu T=k_{T}$ for each $T \in \mathcal{J}$.

In particular, there is a finitely additive extension of Lebesgue measure in the plane which is defined for every set of finite outer measure and multiplies properly under every similarity transformation.

4. Compact groups. In this section we complete the proof of (3.1), but we must first develop some tools. (4.1) collects some well-known facts. (4.2) follows from a theorem of Smulian [8], but is proved here for the sake of completeness.

(4.1) If $E$ is a Banach space and $F \in E^{* *}$, there is at most one point $y_{F} \in E$ such that $F(f)=f\left(y_{F}\right)$ for each $f \in E^{*}$. If $E$ is finite-dimensional, the point $y_{F}$ exists for each $F \in E^{* *}$. If $T$ is a bounded linear transformation of $E$ into $E$, $F \in E^{* *}, y_{F}$ exists, and $H \in E^{* *}$ is defined by $H(f)=F(f T)\left(f \in E^{*}\right)$, then $y_{H}$ exists and $y_{H}=T y_{F}$.

(4.2) Suppose $E$ is a Banach space, $F \in E^{* *}$, and there is a compact set $X \subset E$ such that $F(f) \leqq \sup _{X} f$ for each $f \in E^{*}$. Then $y_{F}$ exists and $y_{F} \subseteq$ Cl conv $X$. 
Proof. Since $f\left(y_{F}\right)=F(f) \leqq \sup _{X} f, y_{F}$ is included in every closed halfspace containing $X$, and hence $y_{F} \in \mathrm{Cl}$ conv $X$. We still must show that $y_{F}$ exists and for finite-dimensional $E$ this follows from (4.1). Let $\&$ be the set of all finite-dimensional linear subspaces of $E^{*}$, and for each $S \in \&$ let $K_{S}$ be the set of all points $x \in \mathrm{Cl}$ conv $X$ such that $F(f)=f(x)$ for each $f \in S$. We shall show that $K_{\mathrm{S}}$ is non-empty. Since, by a theorem of Mazur [5], Cl conv $X$ is compact, $\left\{K_{S}\right\}$ $S \in \mathscr{S}\}$ must then be a family of compact sets which has the finite intersection property. But then there exists a point $p \in \cap_{S \in \mathscr{S}} K_{S}$ and it is clear that $p=y_{F}$.

We now complete the proof by showing that $K_{S}$ is nonempty for each $S \in \&$ Let

$$
E_{2}=\{x \mid f(x)=0 \text { for each } f \in S\} \text {, }
$$

and let $E_{1}$ be a subspace of $E$ which is complementary to $E_{2}$. Then $E_{1}$ is finitedimensional, and each point $x$ of $E$ has a unique expression in the form $x=x_{1}+$ $x_{2}$ with $x_{i} \in E_{i}$. The map $x_{1} \mid x \in E$ is continuous. For each $f \in S$ let $f_{1}=f \mid E_{1}$. Then the map $f_{1} \mid f \in S$ is an isomorphism of $S$ onto $E_{1}^{*}$. For each $f \in S$ let $G\left(f_{1}\right)=$ $F(f)$. Then $G \in E_{1}^{* *}$, and for each $f_{1} \in E_{1}^{*}$ we have

$$
G\left(f_{1}\right)=F(f) \leqq \sup _{X} f=\sup _{X_{1}} f_{1},
$$

where $X_{1}=\left\{x_{1} \mid x \in X\right\}$. Thus by the finite-dimensional case of (4.2) (already established) there is a point $q_{s} \in \mathrm{Cl}$ conv $\mid X_{1}$ such that $G\left(f_{1}\right)=f_{1}\left(q_{s}\right)$ for each $f_{1} \in E_{1}^{*}$. Since $\mathrm{Cl}$ conv $X$ is compact and $(\operatorname{conv} X)_{1}=\operatorname{conv} X_{1}$, we have $\mathrm{Cl}$ conv $X_{1}=(\mathrm{Cl} \operatorname{conv} X)_{1}$. Thus $q_{s}=p_{S_{1}}$ for some $p_{s} \in \mathrm{Cl} \operatorname{conv} X$, and we have

$$
F(f)=G\left(f_{1}\right)=f_{1}\left(q_{s}\right)=f\left(q_{s}\right)=f\left(p_{s}\right)
$$

for each $f \in S$. Hence $p_{s} \in K_{s}$, and the proof of (4.2) is complete.

(4.3) Suppose $A$ is a Banach algebra and $G$ a compact multiplicative subgroup of $A$. Then there are points $u, v \in \mathrm{Cl} \operatorname{conv} G$ such that $u g=u$ and $g v=v$ for each $g \in G$.

Proof. Let $\mu$ be the right-invariant Haar measure on $G$ with $\mu G=1$. For each $f \in E^{*}$ let $F(f)=\int_{G} f d \mu$. Then $F$ satisfies the conditions of (3.2), so $y_{F}$ exists, with $y_{F} \in \mathrm{Cl}$ conv $G$. Consider an arbitrary $g \in G$. Let $T x=x g$ for each $x \in E$, and let $H$ be defined as in (4.1). Then by (4.1), $y_{H}=T y_{F}$. But

$$
H(f)=F(f T)=\int_{G} f(x g) \mid x d \mu=\int_{G} f d \mu=F(f),
$$

so $H=F$. Hence $T y_{F}=y_{F}$, and since $T y_{F}=y_{F} g$ we see that $y_{F}$ is the desired point $u$. Using left-invariant measure, we obtain the desired $v$. 
If the identity map $m$ of $G$ onto $G$ is regarded as a function from the measurespace $(G, \mu)$ to the Banach space $A$, then $u$ is merely the Pettis integral [7] of $m$.

Now returning to the proof of (3.1), suppose (d) holds. Then applying (4.3) to the Banach algebra of bounded linear transformations of $E$ into $E$, where $E=$ $(L, p)$, we obtain a bounded linear transformation $R$ of $E$ into $E$ such that $R \in$ $\mathrm{Cl}$ conv $\mathcal{J}$ and $R T=R$ for each $T \in \mathcal{J}$. From $R \in \mathrm{Cl}$ conv $\mathcal{J}$ it follows easily that $f R=f$ and $p R(z) \in \mathrm{Cl} p(\operatorname{conv} J(z))$ for each $z \in L$, so (b) holds and the proof of ( 3.1$)$ is complete.

We conclude with:

(4.4) Suppose $K$ is a compact convex subset of the Banach space E and $\mathrm{J}$ is a compact group (in the uniform topology of operators) of bounded linear transformations of $E$ onto $E$, each mapping $K$ into $K$. Then there is a point of $K$ which is invariant under every transformation in $\mathrm{J}$.

Proof. By (4.3) there is an $R \in \mathrm{Cl}$ conv $\mathcal{J}$ such that $T R=R$ for each $T \in \mathcal{J}$. Since $K$ is convex, if follows that $R K \subset K$. Then by the fixed-point theorem of Tychonoff [9], $R$ admits a fixed-point $x \in K$. For each $T \in \mathcal{J}$ we have $T x=$ $T R(x)=R(x)=x$, so the proof is complete.

For related results see Kakutani [4].

\section{References}

1. R. P. Agnew and A. P. Morse, Extensions of linear functionals, with applications to limits, integrals, measures, and densities, Ann. of Math. 39 (1938), 20-30.

2. Stefan Banach, Théorie des opérations linéaires, Warsaw, 1932.

3. N. Dunford, Direct decompositions of Banach spaces, Bol. Soc. Mat. Mexicana. 3 (1946), 1 - 12.

4. Shizuo Kakutani, Two fixed-point theorems concerning bicompact convex sets, Proc. Imp. Acad. Tokyo 14 (1938), 242-245.

5. S. Mazur, Über die kleinste konvexe Mengen, die eine gegebene kompakte Menge enthält, Studia Math. 2 (1930), 7-9.

6. Anthony P. Morse, Squares are normal, Fund. Math. 36 (1949), 35-39.

7. B. J. Pettis, On integration in vector spaces, Trans. Amer. Math. Soc. 44 (1938), 277-304.

8. V. $\breve{S}$ mulian, Sur les espaces lineaires topologiques, II. Rec. Math. [Mat. Sbornik] N.S. $9[51](1941), 727-730$.

9. A. Tychonoff, Ein Fixpunktsatz, Math. Ann. 111 (1935), 767-776. 
10. M. A. Woodbury, Invariant functionals and measures, Bull. Amer. Math. Soc. 56 (1950), 172, Abstract $168 \mathrm{t}$.

11. Bertram Yood, On fixed points for semi-groups of linear operations, Proc. Amer. Math. Soc. 2 (1951), 225-233.

UNIVERSITY OF WASHINGTON 


\title{
PACIFIC JOURNAL OF MATHEMATICS
}

\section{EDITORS}

\author{
M. M. SCHIFFER* \\ Stanf ord University \\ Stanf ord, California \\ E. HewitT \\ University of Washington \\ Seattle 5, Washington
}

\author{
P. P. DILWCR TH \\ California Institute of Technology \\ Pasadena 4, California \\ E. F. BECKENBACH** \\ University of California \\ Los Angeles 24, California
}

\section{ASSOCIATE EDITORS}

$\begin{array}{ll}\text { H. BUSEMANN } & \text { P. R. HALMOS } \\ \text { HERDERT FF.DERER } & \text { HEINZ HOPF } \\ \text { MARSH ALLL IHALL } & \text { R. D. JAMES }\end{array}$

MARSH AL.L II ALL

\author{
R. D. JAMES
}
BORGE JESSEN
PAUL LÉVY
GEORGE PÓLYA

\author{
J. J. STOKER \\ E. G. STRAUS
}
KÖSAKU YOSIDA

\section{SPONSORS}

UNIVERSITY OF BRITISH COLUMBIA CAI IFORNIA ENSTITUTE OF TECHNOLOGY UNIVERSITY OF CALIFORNIA, BERKELEY UNIVERSITY OF CALIFORNIA, DAVIS UNIVERSITY OF CALIFORNIA, LOS ANGELES UNIVERSITY OF CALIFORNI A, SANTA BARBARA UNIVERSITY OF NEVADA OREGON STATE COLLEGE UNIVERSITY OF OREGON
UNIVERSITY OF SOU THERN CALIFORNIA STANFORD RESE.ARCH INSTITUTE STANFORD UNIVERSITY WASHING TON STATE COLLEGE UNIVERSITY OF WASHINGTON

AMERICAN MATHEMATICAL SOCIETY HUGHES AIRCRAFT COMPANY SHELL DEVELOPMENT COMPANY

* To be succeeded in 1955, by H.L. Royden, Stanford University, Stanford, California.

${ }^{* *}$ To be succeeded in 1955, by E.G. Straus, University of California, Los Angeles 24, Calif.

\author{
Vari-Type Composition by \\ Elaine Barth
}

Printed in the United States of America by

Edwards Brothers, Inc., Ann Arbor, Michigan

UNIVERSITY OF CALIFORNIA PRESS * BERKELEY AND LOS ANGELES COPYRIGHT 1954 BY PACIFIC JOURNAL OF MATHEMATICS 


\section{Pacific Journal of Mathematics}

\section{Vol. 4, No. 1 \\ May, 1954}

Hugh D. Brunk, On the growth of functions having poles or zeros on the positive real axis ................................. 1

J. Copping, Application of a theorem of Pólya to the solution of an infinite

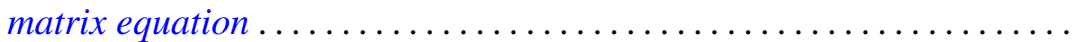

James Richard Jackson, On the existence problem of linear programming ................................... 29

Victor Klee, Invariant extension of linear functionals ............... 37

Shu-Teh Chen Moy, Characterizations of conditional expectation as a transformation on function spaces ..................... 47

Hukukane Nikaidô, On von Neumann's minimax theorem .............. 65

Gordon Marshall Petersen, Methods of summation .................. 73

G. Power, Some perturbed electrostatic fields .................. 79

Murray Harold Protter, The two noncharacteristic problem with data partly

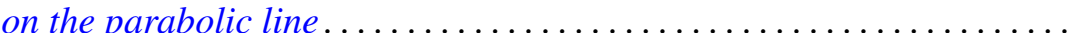

S. E. Rauch, Mapping properties of Cesàro sums of order two of the geometric series........................................... 109

Gerson B. Robison, Invariant integrals over a class of Banach spaces . . . . 123

Richard Steven Varga, Eigenvalues of circulant matrices .............. 151 